\title{
The High-Density Symmetry Energy and Direct Urca
}

\author{
Andrew W. Steiner ${ }^{1}$ \\ ${ }^{1}$ Theoretical Division, Los Alamos National Laboratory, Los Alamos, NM 87545, USA
}

\begin{abstract}
The symmetry energy of nucleonic matter is usually assumed to be quadratic in the isospin density. While this may be justified at sub-saturation densities, there is no need to enforce this restriction at super-saturation densities. The presence of a quartic term can strongly modify the critical density for the direct Urca process which leads to faster cooling of neutron stars. Neutron star cooling predictions which lie below the observational data can, for some equations of state, be repaired with a quartic term which effectively turns off the direct Urca process.
\end{abstract}

PACS numbers: 97.60.Jd, 26.60.+c, 21.65.+f

\section{INTRODUCTION}

The equation of state of nucleonic matter is often decomposed into an isospin-symmetric and an isospin asymmetric part

$$
E(n, \delta)=E_{\text {nuc }}(n, \delta=0)+\delta^{2} S(n)+\delta^{4} Q(n)+\ldots,
$$

where $n=n_{n}+n_{p}, \delta=1-2 n_{p} / n, E_{\text {nuc }}$ is the energy per baryon of nuclear matter and $S$ and $Q$ are arbitrary functions of density. Most often, $Q$ is sufficiently small so that it can be ignored, and then $E_{\text {sym }}=S(n)$ is the densitydependent and isospin-independent symmetry energy.

Without the Coulomb interaction, the near isospin symmetry of QCD predicts that pure neutron matter and pure proton matter should have the same energy density. Terms in Eq. 11 with odd-powers in delta are thus forbidden, assuming the $\delta$ expansion is analytic. Near the nuclear saturation density, it has been suggested that the truncation to order $\delta^{2}$ for the potential energy part of the equation of state is a very good approximation for densities below or near the nuclear saturation density, 0.16 $\mathrm{fm}^{-3}$ 1, 2, 3, 4, 5, 6]. In this article, the effect of the quartic term in Eq. 1 at higher density is examined, where no constraints on the quartic terms are known because the uncertainty contained in many-body effects.

The major effect of a "quartic term" (i.e. a term that is of fourth-order in the isospin deviation, $\delta$ ) is to change the ratio of protons to neutrons at higher densities. All other things remaining equal, the effect on neutron star masses and radii from a quartic term is typically small. However, the critical density for the direct Urca cooling process [7],

$$
n \rightarrow p+e+\bar{\nu}_{e} \text { and } p+e \rightarrow n+\nu_{e}
$$

is very sensitive to the number of protons present in dense matter. This process can only proceed when the conditions for momentum and energy conservation can be simultaneously satisfied, when the proton fraction is larger than about 10 percent. The presence of a quartic term in Eq. 11 can either move the critical density for the direct Urca process to much lower densities, or to densities larger than the central density of the maximum mass neutron star.
Neutron stars cool much more quickly when the direct Urca process is allowed [8, 9]. Thus understanding the possible magnitude of quartic terms is essential in describing the cooling of neutron stars.

\section{ORIGINS OF QUARTIC TERMS}

For the present discussion, it is useful to separate the equation of state into contributions to the potential and kinetic energy. While this demarcation is not necessarily unique, we use the term "kinetic energy part" to refer to the Fermi gas portion (including the interactions present in the nucleon effective mass) of the equation of state (whether relativistic or non-relativistic) and "potential energy part" to refer to the remainder. The kinetic energy part of the symmetry energy naturally contains quartic terms in all equations of state. The potential energy part of the equation of state is the primary consideration here, but it should be noted that interactions can affect the size of quartic terms in the kinetic energy through their effect on the nucleon effective masses.

In relativistic mean-field models 10, 11, 12, 13, 14] the many-body interactions have a non-trivial isospin dependence even in their most trivial form. The linear Walecka model, for example, already has quartic terms (albeit small) in the potential part of the energy density. These quartic terms can be much stronger when three- and higher-body contributions are included. An example is a term proportional to $\sigma \rho^{2}$ where $\sigma$ is the scalar-isoscalar meson and $\rho$ is the vector-isovector meson. In the context of a relativistic point-coupling model 15, 16, 17], such a term would correspond to a three-body nucleon-nucleon interaction of the form

$$
\bar{\psi} \psi\left(\bar{\psi} \gamma^{\mu} \vec{\tau} \psi\right) \cdot\left(\bar{\psi} \gamma_{\mu} \vec{\tau} \psi\right)
$$

In Skyrme-like models, no quartic term is present in the potential energy part of the equation of state. The two-body interaction present in the Skyrme model results in two powers of density, and the three-body interaction is treated as a density-dependent two-body interaction where the additional density dependence is assumed to be exactly isospin symmetric. 
Modern microscopic-macroscopic [18] and HartreeFock-based [19] models of nuclear masses contain explicit non-quadratic terms in the form of the phenomenological term called the Wigner energy. This term is often thought to originate in isovector pairing 20, 21, 22, 23, 24, 25]. Pairing is thought to be primarily a surface effect and vanishes in the infinite baryon number limit. Thus it does not contribute to the equation of state of infinite nucleonic matter here. However, if the Wigner energy was shown to have a different origin, or if the pairing energy was shown to be partially a volume effect (rather than a surface effect), then it would contribute to the isospin dependence of the symmetry energy. Neutron star matter, however, is typically sufficiently neutron-rich that isovector pairing is not a strong contribution.

\section{CONVENIENT PARAMETERIZATION OF THE QUARTIC DEPENDENCE}

A standard alternative to Eq. 1 is to define the symmetry energy using the second derivative with respect to the isospin asymmetry

$$
E_{\mathrm{sym}}(n, \delta)=\frac{1}{2} \frac{d^{2} E(n, \delta)}{d \delta^{2}}=S(n)+6 Q(n) \delta^{2},
$$

where $\varepsilon$ is the energy density. In this work, Eq. 4 will be taken to be the definition of the symmetry energy. If there are no quartic terms in Eq. 10 then $E_{\mathrm{sym}}=S(n)$ is independent of the isospin asymmetry, $\delta$. In the presence of quartic terms, this definition of the symmetry energy in Eq. 4 is more closely connected to the experimental observables based on nuclei which like close to the $N=Z$ line than the alternative of defining the symmetry energy as the energy difference between pure neutron matter and isospin-symmetric nuclear matter.

A convenient way to parameterize the addition of a quartic term is to define $\eta$ as

$$
\eta(n)=\frac{E(n, 1)-E(n, 1 / 2)}{3[E(n, 1 / 2)-E(n, 0)]}=\frac{4 S(n)+5 Q(n)}{4 S(n)+Q(n)} .
$$

This parameter is unity when the quartic term is zero and greater or less than one depending on whether the symmetry energy is increased or decreased by the quartic term. This parameter is more convenient for numerical work than using the generalization of Eq. 目 [6],

$$
E_{\mathrm{sym}, 4}(n)=\frac{1}{24} \frac{d^{4} E(n, \delta)}{d \delta^{4}}=Q(n),
$$

since numerical fourth derivatives can be difficult to compute accurately. The value of $\eta$ is restricted by ensuring that the derivative of the energy per baryon as a function of delta does not vanish at any point other than at $\delta=0$ corresponding to isospin-symmetric nuclear matter. This guarantees that, at fixed density, nuclear matter is always the most energetically favored configuration and neutron matter is always the most costly configuration (when ignoring the Coulomb interaction and possible constraints from $\beta$-equilibrium). The proper limits on $\eta, 3 / 7<\eta<5$ are easily obtained from the definition above. Sometimes it is useful to distinguish the value of $\eta$ as obtained from the "kinetic" part of the EOS, $\eta_{\text {kin }}$, that obtained from the "potential" part of the EOS, $\eta_{\text {pot }}$, and that obtained from the full EOS, $\eta_{\text {tot }}$. Note that $\eta_{\text {tot }}$ is not trivially related to the individual contributions $\eta_{\text {kin }}$ and $\eta_{\text {pot }}$.

Enforcing a particular density-dependence of $\eta$ on the potential part of a given equation of state is straightforward. Defining $\mathcal{T}$ as

$$
\begin{aligned}
\mathcal{T}\left(x_{p}\right)= & \frac{4}{1+3 \eta}\left[-3 x_{p}+19 x_{p}^{2}-32 x_{p}^{3}+16 x_{p}^{4}+\right. \\
& \left.7 x_{p} \eta-23 x_{p}^{2} \eta+32 x_{p}^{3} \eta-16 x_{p}^{4} \eta\right],
\end{aligned}
$$

where $x_{p}=n_{p} / n$, one can construct a new equation of state from any equation of state of neutron and nuclear matter using

$$
E_{\text {pot }}(n, \delta)=\mathcal{T} E_{\text {pot,nuc }}(n)+(1-\mathcal{T}) E_{\text {pot,neut }}(n) .
$$

(Note that the limit $\eta \rightarrow 1$ gives the correct expression for a purely quadratic symmetry energy, $1-\left(1-2 x_{p}\right)^{2}$.) Only the potential energy part of the equation of state has been modified, and the kinetic energy part remains unchanged. The quantity $\mathcal{T}$ is density dependent if $\eta$ is density dependent, as is typical for many equations of state. The corresponding potential parts of the neutron and proton chemical potentials are given by

$$
\begin{aligned}
\mu_{\mathrm{pot}, n}= & \mathcal{T} \mu_{\mathrm{pot}, \text { nuc }, n}+(1-\mathcal{T}) \mu_{\mathrm{pot}, \text { neut }, n}+ \\
& T^{\prime}\left(x_{p}\right) \frac{\left(x_{p}-1\right)}{n}\left(\mu_{\mathrm{pot}, \text { neut }, n}-\mu_{\mathrm{pot}, \mathrm{nuc}, n}\right) \\
\mu_{\mathrm{pot}, p}= & \mathcal{T} \mu_{\mathrm{pot}, \text { nuc }, p}+(1-\mathcal{T}) \mu_{\mathrm{pot}, \text { neut }, p}+ \\
& T^{\prime}\left(x_{p}\right) \frac{x_{p}}{n}\left(\mu_{\mathrm{pot}, \text { neut }, p}-\mu_{\mathrm{pot}, \text { nuc }, p}\right)
\end{aligned}
$$

The effective masses are not modified here since they are in the kinetic energy part of the EOS.

The behavior of the energy per baryon as a function of $\delta$ at saturation density in the equation of state (EOS) of Akmal, et. al (APR). 26] constructed as above to have varying values of $\eta_{\text {pot }}$ is given in Fig. 10 The deviation is not large, at most a couple $\mathrm{MeV}$ near saturation density for the most extreme values of $\eta$.

\section{THE VALUE OF $\eta$ FOR MODERN EQUATIONS OF STATE}

In addition to APR from above, several relativistic mean-field (RMF) models are used in this work, all of which are constrained to have the following properties at saturation

$$
\begin{aligned}
\mathrm{n}_{0} & =0.16 \mathrm{fm}^{-3} \\
\mathrm{E} / \mathrm{A} & =-16 \mathrm{MeV}
\end{aligned}
$$




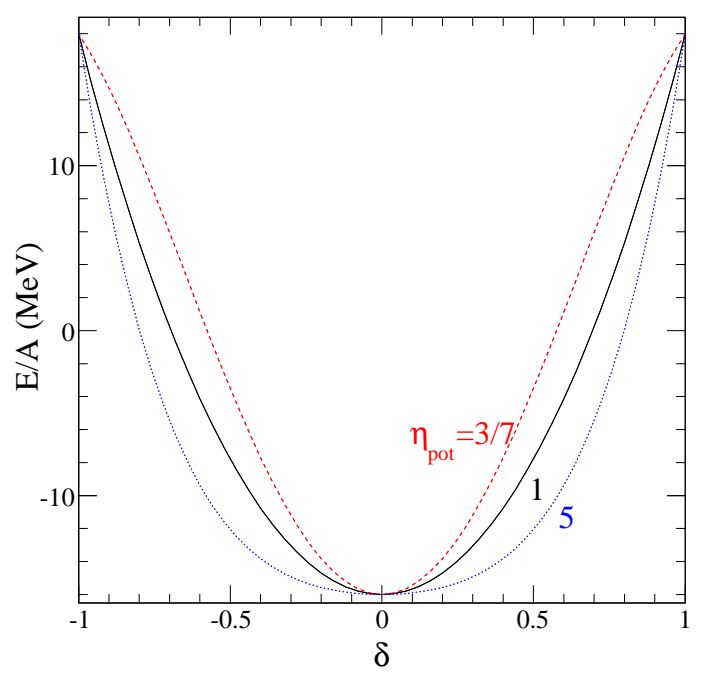

FIG. 1: The energy per baryon of the APR EOS at saturation density as a function of the proton fraction, $x_{p}$, for the trivial and limiting values of $\eta_{\text {pot }}$.

$$
\begin{aligned}
\mathrm{K} & =230 \mathrm{MeV} \\
\mathrm{E}_{\mathrm{sym}} & =34 \mathrm{MeV} \\
\mathrm{M}^{*} / \mathrm{M} & =0.8
\end{aligned}
$$

where $\mathrm{n}_{0}$ is the saturation density, $\mathrm{E} / \mathrm{A}$ is the binding energy, $\mathrm{K}$ is the compressibility, $\mathrm{E}_{\mathrm{sym}}$ is the symmetry energy at the saturation density, and $\mathrm{M}^{*} / \mathrm{M}$ is the reduced Dirac effective mass. In addition to the standard RMF model with no non-linear couplings except for the cubic and quartic self-interactions among the scalar mesons, two RMF models are constructed with small ("RMFlo") and large ("RMFhi") values of $\eta_{\text {pot }}$ in order to demonstrate the variations that are possible within this formalism. These two models do not necessarily represent the most extreme cases, as a full optimization was not performed. The couplings are given in Table 1 and utilize the Lagrangian and notation described in [27]. All of these couplings are within a factor of two of the constraints required by naturalness [13, 28].

The Skyrme 29] model "SLy230a" [30] is used for comparison. This model matches the binding energies and charge radii of several ground-state nuclei, the known properties of saturated nuclear matter, and results in reasonable neutron star properties.

The momentum dependence of the nucleon optical potential is essential for transport simulations of heavy-ion collisions. The MDI (momentum-dependent interaction) EOS [31], which has successfully described aspects of heavy-ion collisions at intermediate energies, is included here. In addition to providing for reasonable properties of saturated nuclear matter, the isoscalar potential of the MDI EOS coincides with predictions from the variational many-body theory using inputs constrained by nucleonnucleon scattering data 3, 32], and the isovector potential agrees with the momentum dependence of the Lane
TABLE I: The couplings for the RMF, RMFlo, and RMFhi models. The units are all given so that the Lagrangian has

\begin{tabular}{|c|c|c|c|}
\hline \multicolumn{4}{|c|}{$\mathrm{RMF}$} \\
\hline$g_{\sigma}$ & 7.721 & $g_{\omega}$ & 7.955 \\
\hline$g_{\rho}$ & 8.608 & $\kappa$ & 21.40 \\
\hline$\lambda$ & $-6.227 \times 10^{-4}$ & & \\
\hline \multicolumn{4}{|c|}{ RMFlo } \\
\hline$g_{\sigma}$ & 7.692 & $g_{\omega}$ & 7.998 \\
\hline$g_{\rho}$ & 14.00 & $\kappa$ & 18.9 \\
\hline$\lambda$ & $5.188 \times 10^{-3}$ & $\zeta$ & 0.03801 \\
\hline$\xi$ & 1.499 & $a_{1}$ & 69.6 \\
\hline$a_{2}$ & 1.052 & $b_{1}$ & 0.05251 \\
\hline$a_{3}$ & $8.446 \times 10^{-3}$ & $a_{4}$ & $-2.063 \times 10^{-5}$ \\
\hline$b_{2}$ & $-8.085 \times 10^{-6}$ & & \\
\hline \multicolumn{4}{|c|}{ RMFhi } \\
\hline$g_{\sigma}$ & 7.677 & $g_{\omega}$ & 8.021 \\
\hline$g_{\rho}$ & 7.551 & $\kappa$ & 17.50 \\
\hline$\lambda$ & $8.361 \times 10^{-3}$ & $\zeta$ & 0.05880 \\
\hline$\xi$ & 0.02870 & $a_{1}$ & -35.30 \\
\hline$a_{2}$ & -0.66605 & $b_{1}$ & 0.2719 \\
\hline$a_{3}$ & $1.281 \times 10^{-3}$ & $a_{4}$ & $2.062 \times 10^{-5}$ \\
\hline$b_{2}$ & $1.127 \times 10^{-5}$ & & \\
\hline
\end{tabular}
units $\mathrm{MeV}^{4}$ when the meson fields are in $\mathrm{MeV}$ and the nucleon fields in $\mathrm{MeV}^{3 / 2}$. Omitted couplings are equal to zero.

potential extracted from $(\mathrm{p}, \mathrm{n})$ charge exchange reactions up to about $45 \mathrm{MeV}$ 32, 33. We use the formulation as described in Ref. 31] with the symmetry energy parameter, $x$, set equal to zero.

The APR and Skyrme EOSs are special in that $\eta_{\text {pot }}=$ 1 independent of density. In the case of the Skyrme models, this results from the structure of the interaction, and in the case of APR, $\eta_{\text {pot }}=1$ by construction.

In Fig. 2] the intrinsic values of $\eta$ (the procedure described in Eqs. 7.9 has not yet been employed) as obtained from the EOSs described above are plotted, as well as that obtained by fitting the results in Ref. [5]. Note that only the total energy per baryon was reported in this latter reference, so there are no results for the top panel. The value of $\eta_{\text {pot }}$ for the typical RMF model is quite close to unity, while RMFlo and RMFhi (by construction) have values significantly different from 1 . The maximum value of $\eta_{\text {pot }}$ for the RMFhi EOS, even though it lies outside the graph, is below the limit $\eta<5$ mentioned in the introduction. The MDI EOS has a large value of $\eta_{\text {pot }}$, even at lower densities where most models have $\eta_{\text {pot }} \sim 1$

The values of $\eta_{\text {tot }}$ shown in the bottom panel show a little more variation. The value of $\eta_{\text {tot }}$ from the BHF calculation is quite close to 1 , even at lower densities where other models (except RMFlo) predict larger values. This is typical; in order to get $\eta_{\text {tot }}$ equal to unity at low densities, one frequently has $\eta_{\text {pot }}<1$ in order to cancel the 
effect of $\eta_{\text {kin }}$ which is most often larger than one. It is also quite clear that the effects of the kinetic part of the equation of state are not trivial in determining $\eta_{\text {tot }}$. The ordering of the values of $\eta$ for the RMFlo and RMFhi EOSs reverses depending on whether or not one is considering the total equation of state or only the potential energy part.

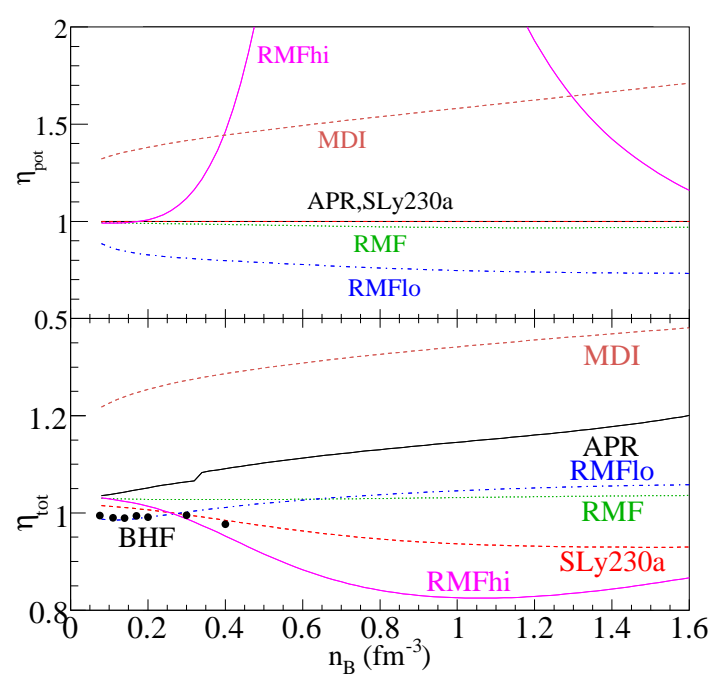

FIG. 2: The density dependence of $\eta_{\text {pot }}$ and $\eta_{\text {tot }}$ determined from Eq. 5 for the equations of state considered in this work as described in the text. The kink in APR is due to the phase transition to the high-density phase.

\section{DIRECT URCA}

When the neutron star temperature is small enough that it can be ignored in comparison to the Fermi momenta of the consituents, the critical density for direct Urca is equivalent to the condition that one be able to form a triangle with the neutron, proton, and electron Fermi momenta. This triangle may be formed if the squared area obtained by Heron's formula is positive, i.e.

$$
s\left(s-k_{F n}\right)\left(s-k_{F p}\right)\left(s-k_{F e}\right)>0
$$

where $s$ is the semiperimeter defined by $s=\left(k_{F n}+\right.$ $\left.k_{F p}+k_{F e}\right) / 2$. This reduces the familiar condition $k_{F n}<$ $k_{F p}+k_{F e}$ for neutron-rich matter and is more easily generalizable to other Urca-like processes.

When an EOS has a proton fraction which is small, true of the APR EOS and some EOSs based on Skyrme interactions, the presence of a quartic term can drastically affect the critical density for the direct Urca process. This is demonstrated in Fig. 3] where the critical density is plotted as a function of $\eta_{\text {pot }}$, assuming that $\eta_{\text {pot }}$ is density independent. Depending on the relative strength of the quartic dependence, Urca either proceeds in all neutron stars with masses larger than about 1.4 solar masses or in no neutron stars of any mass. Although values of $\eta_{\text {pot }}$ less than $1 / 2$ are allowed, the corresponding critical densities are already above the central density for the maximum mass configuration. The maximum mass and radius of the maximum mass neutron star as a function of $\eta_{\text {pot }}$ are also plotted in Fig. 3. These quantities are essentially unaffected by the modification of $\eta_{\text {pot }}$.

RMF models typically have a large symmetry energy and a large proton fraction, and thus allow the direct Urca process at low densities. However, this is not required, and Ref. 27] constructed a couple RMF models with small neutron star radii (named SR1, SR2, and SR3) which do not allow direct Urca at any density lower than the central density of the maximum mass configuration. These models also typically have a smaller symmetry energy at saturation density than the models utilized here. The models RMF, RMFlo, and RMFhi, have critical densities of $0.306,0.275$, and $0.806 \mathrm{fm}^{-3}$, respectively. Note that RMFhi has a rather large critical density for direct Urca which could easily be made larger by decreasing the value of the symmetry energy at saturation density.

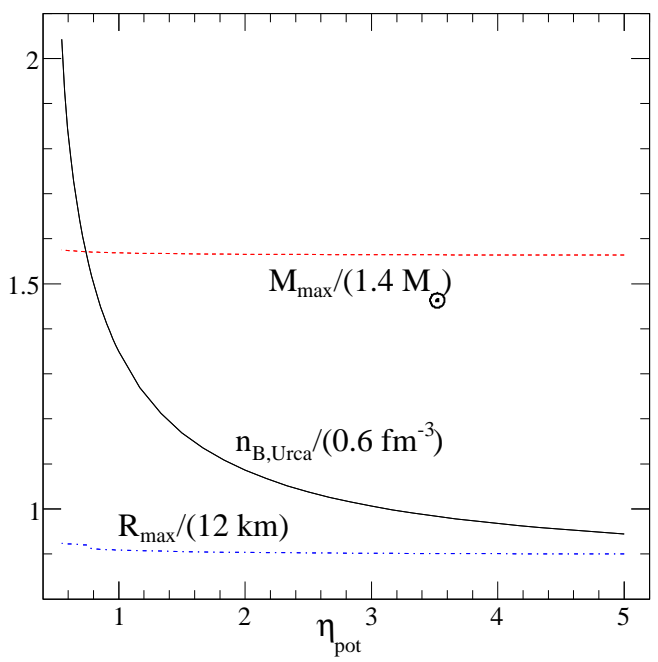

FIG. 3: The critical density for the direct Urca process for the APR EOS as a function of $\eta_{\text {pot }}$. Points with $\eta_{\text {pot }}<1 / 2$ were not plotted because the Urca process is not allowed at any density for this range of $\eta$.

Ref. 34] compared the radii of $1.4 \mathrm{M}_{\odot}$ neutron stars with the MDI EOS using different symmetry energies and comparing with isospin diffusion data from intermediateenergy heavy-ion collisions. This reference found that the threshold for the direct URCA process was signficantly different for the APR EOS and for the MDI EOS (with $x=0$ ) even though they had nearly identical symmetry energies. This is, in large part, due to the signficant presence of quartic terms in the MDI EOS as demonstrated above. This does not contradict the constraint on neutron star radii from Ref. 34], since neutron star radii are insensitive to quartic terms, as demonstrated in Fig. 3. 


\section{DISCUSSION}

Quartic terms play an important role in determining the critical density for the direct Urca process in neutron stars. These terms can be easily generated within the context of RMF models of high-density nucleonic matter. While this work means that it will be more difficult to interpret neutron star cooling data without more information on the value of $\eta$ at large densities, it also means that more neutron star cooling data is essential to understanding the nature of the high-density equation of state. Observations of neutron stars masses and radii will have difficulty constraining the value of $\eta$.

Ref. 35] studied in detail the cooling of neutron stars constructed with the APR EOS. They found that, because of the direct Urca process, stars with masses larger than about $1.7 \mathrm{M}_{\odot}$ cool sufficiently rapidly as to be cooler than nearly all of the observed neutron stars. As these authors point out, this is somewhat sensitive to the assumptions about the pairing interaction, and sufficiently cool neutron stars with large mass may be difficult to obeserve. Nevertheless, this work offers another possible interpretation: the actual value of $\eta_{\text {pot }}$ may be sufficiently small at high density to turn off the direct Urca process thus making the computed cooling curves match the comparatively warm neutron stars which are given in the experimental data.

Proto-neutron star cooling (during the first minute when the core is still hot) always contains a significant contribution from direct Urca (e.g. see the review in Ref. [36]) since the finite temperature allows momentum conservation to be easily fulfilled. However, the proton fraction of the hot neutron star matter dictates the flavor content of the associated neutrino signal and this will be modified by the presence of quartic terms.

Ref. 27] pointed out that there is a correlation between the threshold for direct Urca and the neutron skin thickness of lead. This work clearly indicates that the correlation is predicated on the implicit assumption that $\eta_{\text {pot }}$ is nearly unity for the relevant densities. This will also, for some models, impact the suggested correlation between the direct Urca process and neutron star radii [37], since quartic terms are important for the former but not for the latter.

It might be interesting to explore the effect of a quartic term on the symmetry energy at finite temperature. The value of $\eta$ for the kinetic part of the equation of state could become closer to unity since finite-temperature effects are proportional to $T^{2}$. Nevertheless, various experiments are now probing the temperature-dependence of the symmetry energy and might provide constraints on a quartic behavior [38, 39, 40].

The effects of a phase transition to hyperons, Bosecondensates (except for that already present in APR), or quarks have been ignored. It might be interesting to explore the value of $\eta$ for equations of state involving phase transitions. Although quark matter is often more isospinsymmetric, it also frequently has a significant gap, which prohibits the direct Urca process for low enough temperatures.

\section{ACKNOWLEDGEMENTS}

The author would like to thank Bao-An Li, Peter Möller, Madappa Prakash, and Sanjay Reddy for their helpful comments. This work was carried out under the auspices of the National Nuclear Security Administration of the U.S. Department of Energy at Los Alamos National Laboratory under Contract No. DE-AC5206NA25396.
[1] I. E. Largaris and V. R. Pandharipande, Nucl. Phys. A 369, 470 (1981).

[2] M. Prakash and T. L. Ainsworth, Phys. Rev. C 36, 346 (1987).

[3] R. B. Wiringa, V. Fiks, and A. Fabrocini, Phys. Rev. C 38, 1010 (1988).

[4] H. Müther, M. Prakash, and T. L. Ainsworth, Phys. Lett. B 199, 469 (1988).

[5] I. Bombaci and U. Lombardo, Phys. Rev. C 44, 1892 (1991).

[6] C.-H. Lee, T. T. S. Kuo, G. Q. Li, and G. E. Brown, Phys. Rev. C 57, 3488 (1998).

[7] J. M. Lattimer, C. J. Pethick, M. Prakash, and P. Haensel, Phys. Rev. Lett. 66, 2701 (1991).

[8] D. Page and J. H. Applegate, Astrophys. J. Lett. 394, 17 (1992).

[9] J. M. Lattimer, K. A. van Riper, M. Prakash, and M. Prakash, Astrophys. J. 426, 802 (1994).

[10] J. D. Walecka, Ann. Phys. (NY) 83, 491 (1974).

[11] J. Boguta and A. R. Bodmer, Nucl. Phys. A 292, 413
(1977).

[12] B. D. Serot, Phys. Lett. B 86, 146 (1979).

[13] H. Müller and B. D. Serot, Nucl. Phys. A 606, 508 (1996).

[14] R. Furnstahl, Nucl. Phys. A 706, 85 (2002).

[15] J. J. Rusnak and R. J. Furnstahl, Nucl. Phys. A 627, 495 (1997).

[16] B. A. Nikolaus, T. Hoch, and D. G. Madland, Phys. Rev. C 56, 177 (1997).

[17] T. Bürvench, D. G. Madland, J. A. Maruhn, and P.-G. Reinhard, Phys. Rev. C 65, 044308 (2002).

[18] P. Moller, J. R. Nix, W. D. Myers, and W. J. Swiatecki, At. Data. Nucl. Data Tables 59, 185 (1995).

[19] M. Samyn, S. Goriely, P.-H. Heenen, J. M. Pearson, and F. Tondeur, Nucl. Phys. A 700, 142 (2002).

[20] W. Satuła and R. Wyss, Phys. Lett. B 393, 1 (1997).

[21] W. Satuła, D. J. Dean, J. Gary, S. Mizutori, and W. Nazarewicz, Phys. Lett. B 407, 103 (1997).

[22] W. Satuła, J. Dobaczewski, and W. Nazarewicz, Phys. Rev. Lett. 81, 3599 (1998).

[23] P. Vogel, Nucl. Phys. A 662, 148 (2000). 
[24] A. V. Afanasjev and S. Frauendorf, Phys. Rev. C 71, 064318 (2005).

[25] A. O. Macchiavelli, P. Fallon, R. M. Clark, M. Cromaz, M. A. Deleplanque, R. M. Diamond, G. J. Lane, I. Y. Lee, F. S. Stephens, C. E. Svensson, et al., Phys. Rev. C. 61, 041303(R) (2000).

[26] A. Akmal, V. R. Pandharipande, and D. G. Ravenhall, Phys. Rev. C 58, 1804 (1998).

[27] A. W. Steiner, M. Prakash, J. M. Lattimer, and P. J. Ellis, Phys. Rep. 411, 325 (2005).

[28] J. Friar, D. G. Madland, and B. W. Lynn, Phys. Rev. C 53, 3085 (1996).

[29] T. H. R. Skyrme, Nucl. Phys. 9, 615 (1959).

[30] E. Chabanat, P. Bonche, P. Haensel, J. Meyer, and R. Schaeffer, Nucl. Phys. A627, 710 (1997).

[31] C. B. Das, S. Das Gupta, C. Gale, and B.-A. Li, Phys. Rev. C 67, 034611 (2003).

[32] B.-A. Li, L.-W. Chen, C. B. Das, S. Das Gupta, C. Gale,
G. C. Yong, and W. Zuo, nucl-th/0504069 (2005).

[33] G. Hoffmann, Phys. Rev. Lett. 29, 227 (1972).

[34] B.-A. Li and A. W. Steiner, nucl-th/0511064 (2005).

[35] M. E. Gusakov, A. D. Kaminker, D. G. Yakovlev, and O. Y. Gnedin, Mon. Not. R. Astron. Soc. 363, 555 (2005).

[36] M. Prakash, I. Bombaci, M. Prakash, P. J. Ellis, and J. M. Lattimer, Phys. Rep. 280, 1 (1997).

[37] C. J. Horowitz and J. Piekarewicz, Phys. Rev. C 66, 055803 (2002).

[38] M. B. Tsang, W. A. Friedman, C. K. Gelbke, W. G. Lynch, G. Verde, and H. S. Xu, Phys. Rev. Lett. 86, 5023 (2001).

[39] A. Ono, P. Danielewicz, W. A. Friedman, W. G. Lynch, and M. B. Tsang, Phys. Rev. C 68, 051601(R) (2003).

[40] S. Kowalski, J. B. Natowitz, S. Shlomo, R. Wada, K. Hagel, J. Wang, T. Materna, Z. Chen, Y. G. Ma, L. Qin, et al., nucl-ex/0602023 (2006). 